\title{
The construction of thermodynamic expressions needed in the physicochemical mathematical model required for the rational physical and quantitative evaluation of the freeze drying process of pharmaceutical solutions employing tertiary butyl alcohol as a co-solvent
}

\author{
Wang, J. C. ${ }^{\text {a }}$; Bruttini, R. ${ }^{\text {b; Liapis, A. I. }}{ }^{a^{*}}$ \\ ${ }^{a}$ Department of Chemical and Biochemical Engineering, Missouri University of Science and \\ Technology, 110 Bertelsmeyer Hall, 1101 North State Street, Rolla, Missouri 65409-1230, USA \\ b Criofarma - Freeze Drying Equipment, Strada del Francese 97/2L, 10156 Turin, Italy. \\ *E-mail of the corresponding author: ail@mst.edu
}

\begin{abstract}
A thermodynamic model employing the UNIFAC (Dortmund) method was developed to determine the currently unavailable partial vapor pressures of the binary gas mixture of water and tert-butyl alcohol (TBA) in equilibrium with their frozen solid mixtures. The results agree satisfactorily with the experimental data and indicate that TBA has higher vapor pressures which lead to higher total pressures at the moving interface that could result in larger total pressure gradients and convective mass transfer rates in the dried layer during primary drying. But the higher total pressures reduce the magnitude of the bulk diffusivity of the gas mixture and combined with the smaller Knudsen diffusivity of TBA could significantly impact the competing mass transfer mechanisms during freeze drying.
\end{abstract}

Keywords: Freeze drying; Water and tert-butyl alcohol (TBA); UNIFAC (Dortmund); Partial vapor pressures; Convective flow and bulk and Knudsen diffusion 
Thermodynamic expressions for the evaluation of the freeze drying process of pharmaceutical solutions employing tertiary butyl alcohol as a co-solvent

\section{Introduction}

The presence of organic solvents as co-solvents with water in pharmaceutical and biological solutions could reduce the degradation rate of the pharmaceutical/biological product in water and/or increase its solubility. It has been shown experimentally [1] to affect the freezing characteristics of the solution during the freezing stage as well as during the primary and secondary stages of the freeze drying process. In the production of a number of anti-cancer drugs, the tertiary butyl alcohol (TBA, or 2-methyl-2-propanol) represents the organic co-solvent in the water solutions with varying concentrations dependent on the biotechnology method of production and the nature of the anti-cancer drug. Currently due to strict quality and toxicity standards required by the Food and Drug Administration (FDA), most of TBA is removed prior to the freeze drying of the drug solution. In order to ascertain if this is the only or best strategy, it is of paramount importance to acquire both physical and quantitative knowledge of the unsteady state variations and their interactions that occur during the freeze drying process in the (i) temperature and heat transfer rates in the frozen and porous dried layers of the product, (ii) mass transfer mechanisms and rates in the porous dried layer, (iii) relative residual amounts of frozen water and TBA in the frozen solid layer which affect the partial pressures of the vapors of water and TBA at the moving interface separating the frozen and porous dried layers during the primary drying stage, and (iv) the amounts of sorbed (bound) water and TBA on the surface of the pores of the dried layer during the primary and secondary drying stages. Because these data cannot be measured experimentally during the freeze drying process, it becomes necessary to construct and solve an unsteady-state and multidimensional in space mathematical model of the freeze drying process in order to determine, evaluate, and analyze the dynamic behavior of items (i) - (iv) for ascertaining whether or not the presence of TBA in the drug solution could reduce the drying times of the primary and secondary drying stages of the freeze drying process and, furthermore, whether the presence and distribution of the residual TBA in the three-dimensional space of the product being freeze dried could result in the violation of the strict quality and toxicity standards required by the FDA for the freeze drying of pharmaceuticals.

The mathematical model required to be constructed and solved for the freeze drying process of frozen solutions of pharmaceuticals involving water and an organic co-solvent like TBA has now to consider the vapor pressures and mass transfer rates of a ternary gas mixture (inert gas, water, and TBA) in the porous dried layer of the product, which is more complex than the models [2-6] for a binary mixture (inert gas and water) system. The off-diagonal terms of the diffusivity tensor for the three species will require careful examination of the relative concentrations of water and TBA vapors in the pore structure of the dried layer at different temperatures, in order to ascertain whether or not these off-diagonal terms and their effects on the heat and mass transfer processes need to be included in the structure of the mathematical model. The construction and solution of this new class of freeze drying models and the achievement of its aforementioned purposes require the following two pieces of fundamental physical information: (a) the thermodynamic model whose expressions provide the partial pressures of water and TBA vapors in equilibrium with the binary frozen mixtures of water and TBA as a function of temperature and of the mole 
fractions of water and TBA in the frozen solid phase, and this necessary information also provides the important partial pressures of the vapors of water and TBA at the moving interface separating the frozen and dried layers of the product being freeze dried during the primary drying stage of the freeze drying process, and (b) the equilibrium adsorption/desorption isotherms of the sorbed (bound) binary mixtures of water and TBA on the surface of the pores of the dried layer which provide the concentrations of water and TBA in the binary sorbed (bound) mixture as a function of the temperature and of the partial vapor pressures of water and TBA in the gas phase in the pores of the dried layer, and this provides in the mathematical model the quantitative expressions needed to represent binary competitive adsorption/desorption through which the residual amounts of sorbed water and TBA as well as their distributions in the three-dimensional space of the dried layer of the product at any given time and especially at the end of the secondary drying of the freeze drying process could be calculated, so that we can determine whether the strict quality and toxicity criteria of the FDA are properly satisfied.

In this work the UNIFAC method coupled with the Dortmund parameter values for the subgroups of the binary mixture comprised of the species of water and TBA is employed and a thermodynamic equilibrium model is constructed. This model can provide the partial pressures of the vapors of water and TBA in equilibrium with the frozen solid phase of the binary mixture of frozen water and TBA, as a function of the temperature and of the mole fractions of water and TBA in the binary mixture of the frozen solid phase. The results obtained from the thermodynamic equilibrium model constructed and solved in this work are compared with the experimental results of Kasraian and DeLuca [7] and the agreement between the theoretical and the experimental results is found to be good. Therefore, this work considered the necessary and very important research problem stated in item (a) above and provides a satisfactory solution to this important research problem.

\section{Thermodynamic Models and Methods}

The thermodynamic equilibrium of a species between its pure liquid phase and its frozen mixture can be represented by

$$
\ln \left(x_{i} \gamma_{i}\right) \approx \frac{\Delta H_{\mathrm{tri}}^{f u s}}{R T_{\mathrm{tri}}}\left(1-\frac{T_{\mathrm{tri}}}{T}\right)-\frac{\Delta C_{P}^{s l^{*}}}{R}\left[1-\frac{T_{\mathrm{tri}}}{T}+\ln \left(\frac{T_{\mathrm{tri}}}{T}\right)\right],
$$

where $x_{i}$ and $\gamma_{i}$ are the mole fraction and activity coefficient of species $i$, respectively, and $l^{*}$ represents its subcooled liquid state at a temperature below its triple point $\left(T_{\text {tri }}\right)$. Equation (1) has also been used as a means to predict solid-liquid equilibrium for mixture systems. For the binary frozen mixture of water and TBA, its experimental solid-liquid equilibrium phase diagram as demonstrated by Kasraian and DeLuca [7] is quite complex and has two eutectic points. The water-rich region forming pure solid ice and the TBA-rich region forming pure solid TBA were first read from the phase diagram [7] to estimate $\gamma_{i}$ based on Eq. (1) and compared with those estimated by the modified UNIFAC (Dortmund) group contribution method [8]. For this purpose, the needed triple point and molar heat of fusion at $T_{\text {tri }}\left(\Delta H_{\text {tri }}\right)$ are $273.16 \mathrm{~K}$ and $6004 \mathrm{~J} / \mathrm{mol}$ for water and $298.96 \mathrm{~K}$ and $6700 \mathrm{~J} / \mathrm{mol}$ for TBA (National Institute of Standards and Technology, NIST WebBook), respectively. Within the 
temperature ranges, the heat capacity difference, $\Delta C_{P}^{s l^{*}}=C_{P}^{l^{*}}-C_{P}^{s}$, for water is correlated into $\left(\frac{234.784}{T-229.760}+69.924\right)-(0.1051 T+8.323) \mathrm{J} / \mathrm{mol}-\mathrm{K}, T$ in $\mathrm{K}$, while for TBA it is based on $C_{P}^{l^{*}}=C_{\text {tri }}^{l}=2125.37 \mathrm{~J} / \mathrm{mol}-\mathrm{K}$ (NIST WebBook) and $C_{P}^{s}$ interpolated from the reported experimental data [9]. The activity coefficients are shown in Fig. 1 where the levels of agreement could be considered practically satisfactory, especially for the water-rich region, when there is a lack of both experimental data and an alternative theoretical approach.

Despite their importance to the freeze drying operation as well as to the intended mathematical modeling, the partial pressures of the water and TBA vapors above their frozen binary mixtures appear not to have been measured experimentally or reported in the literature. Instead, only the total pressures of the eutectic mixtures at $90 \mathrm{wt} \% \mathrm{TBA}\left(x_{\mathrm{TBA}}=\right.$ 0.6863) have been measured and correlated [10]. In order to further verify the practical usefulness of the thermodynamic methods and models employed and presented in this work, the UNIFAC (Dortmund) method was applied in the following approach to estimate the total eutectic pressures,

$$
\begin{aligned}
& P_{\mathrm{tot}}=P_{\mathrm{W}}+P_{\mathrm{TBA}}=\gamma_{\mathrm{W}} x_{\mathrm{W}} P_{\mathrm{W}}^{l^{*}}+\gamma_{\mathrm{TBA}}\left(1-x_{\mathrm{W}}\right) P_{\mathrm{TBA}}^{l^{*}} . \\
& P_{\mathrm{W}}^{l^{*}}(\mathrm{~Pa})=612 \exp \left[-\frac{45054}{R}\left(\frac{1}{T}-\frac{1}{273.16}\right)\right], T \text { in } \mathrm{K} . \\
& P_{\mathrm{TBA}}^{l^{*}}(\mathrm{~Pa})=\exp \left(-\frac{5583.5}{T}+27.925\right), T \text { in } \mathrm{K} .
\end{aligned}
$$

It is clear from the results presented in Fig. 2 that the thermodynamic approach presented here is able to produce the eutectic pressures satisfactorily within the ranges measured by experiments. The approach presented here could thus be considered a useful and reasonable one, especially in the absence of experimental thermodynamic data as currently is the case, for estimating the partial pressures of water and TBA vapors at other compositions in equilibrium with their binary frozen solid mixtures.

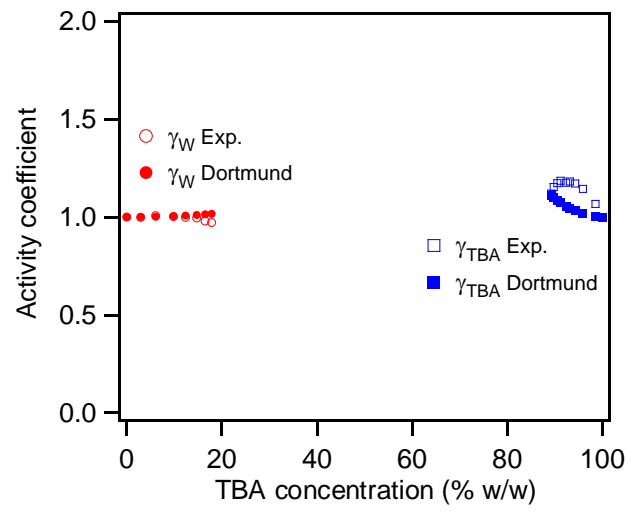

Fig. 1 Activity coefficient as a function of the TBA concentration

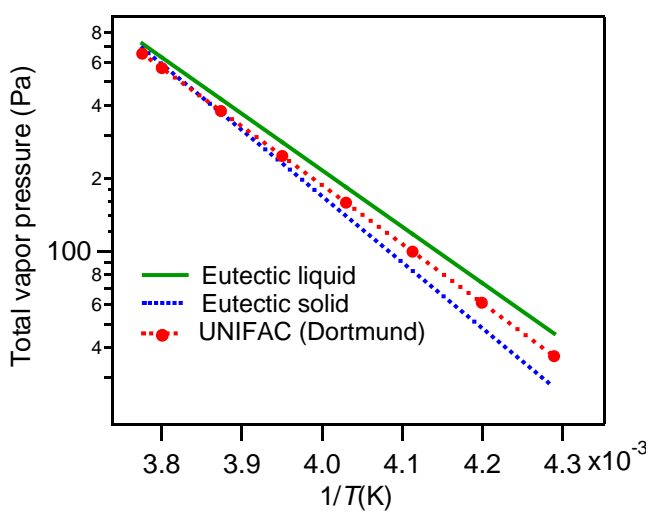

Fig. 2 Total pressure of eutectic mixtures at $x$ TBA $=0.6863$ 
The partial pressures of water and TBA vapors resulting from the approach presented here at different temperatures and water mole fractions in the binary frozen solid phase are plotted in Fig. 3, while the total pressure resulting from adding the two partial pressures together is plotted in Fig. 4. It should be noted that the highest temperature selected to be considered here is $-8.3{ }^{\circ} \mathrm{C}$ because this is the lower eutectic temperature below which the binary mixtures are frozen solids over the entire compositional range $[7,10]$. To incorporate the results of the partial vapor pressures into a future mathematical modeling study of the freeze drying process, well-correlated analytical expressions are much more desirable. For this purpose, only the activity coefficients, as indicated by Eqs. (2a)-(2c), need to be correlated. The functional form of the Non-Random Two-Liquid (NRTL) activity model was found to provide excellent correlations for the partial pressures with the following temperature-dependent parameters where $R$ is the ideal gas constant and $T$ is in $\mathrm{K}$,

$$
\begin{aligned}
& \ln \gamma_{\mathrm{W}}=\ln \left(\frac{P_{\mathrm{W}}}{x_{\mathrm{W}}}\right)=x_{\mathrm{TBA}}^{2}\left[\frac{b_{21}}{R T}\left(\frac{G_{21}}{x_{\mathrm{W}}+x_{\mathrm{TBA}} G_{21}}\right)^{2}+\frac{G_{12} b_{12} / R T}{\left(x_{\mathrm{TBA}}+x_{\mathrm{W}} G_{12}\right)^{2}}\right], \\
& \ln \gamma_{\mathrm{TBA}}=\ln \left(\frac{P_{\mathrm{TBA}}}{1-x_{\mathrm{W}}}\right)=x_{\mathrm{W}}^{2}\left[\frac{b_{12}}{R T}\left(\frac{G_{12}}{x_{\mathrm{TBA}}+x_{\mathrm{W}} G_{12}}\right)^{2}+\frac{G_{21} b_{21} / R T}{\left(x_{\mathrm{W}}+x_{\mathrm{TBA}} G_{21}\right)^{2}}\right], \\
& \left\{\begin{array}{l}
G_{12}=\exp \left(-\alpha \frac{b_{12}}{R T}\right) ;\left\{\begin{array}{l}
\alpha=1.628-0.00992 T+1.860 \times 10^{-5} T^{2} \\
b_{12}(\mathrm{~J} / \mathrm{mol}-\mathrm{K})=-11874.652+101.182 T-0.120 T^{2} \\
b_{21}(\mathrm{~J} / \mathrm{mol}-\mathrm{K})=-7429.529+58.534 T-0.108 T^{2}
\end{array}\right.
\end{array}\right.
\end{aligned}
$$

It is worth noting here that, as pure species or as components with the same mole fractions in the frozen solid mixtures, water has noticeably lower sublimation partial pressures (see Fig. 3) than those of TBA vapor and the resulting total pressure in the porous dried layer of

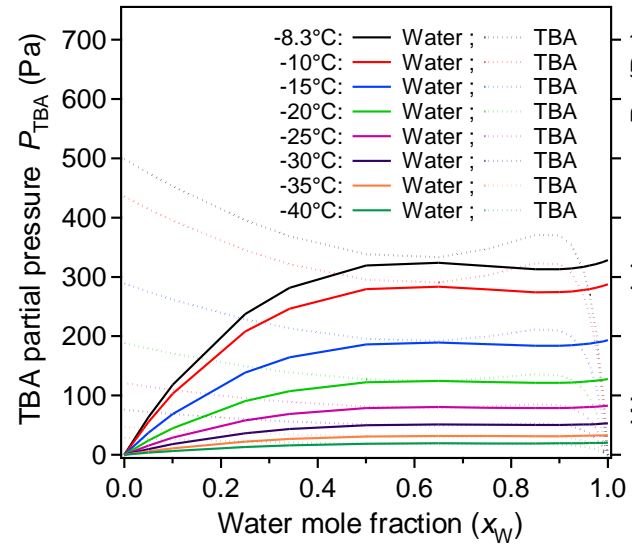

Fig. 3 Partial vapor pressure as a function of temperature and water mole fraction in the binary frozen solid mixture

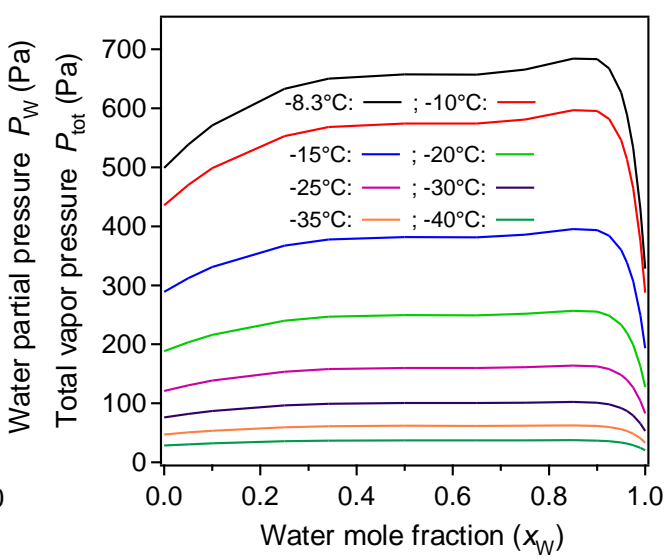

Fig. 4 Total vapor pressure as a function of temperature and water mole fraction in the binary frozen solid mixture 
Thermodynamic expressions for the evaluation of the freeze drying process of pharmaceutical solutions employing tertiary butyl alcohol as a co-solvent

the product being freeze dried is significantly higher than in the case where water was the only solvent and, thus, the resulting total pressure gradient between the moving interface and the drying chamber pressure could be larger and provide an increase in the total convective mass transfer rate of the water and TBA gas mixture due to the presence of TBA. Therefore, TBA could sublime faster during primary drying and facilitate the mass transfer of water vapor from the frozen product and possibly reduce the primary drying time. But there are additionally two diffusion mass transfer mechanisms occurring simultaneously with convective mass transfer in the porous dried layer of the product, namely Knudsen and bulk diffusion. The Knudsen diffusivity is independent of total pressure, but it has dependencies on temperature and molecular mass. Taking a representative pore radius of $1000 \AA$, the Knudsen diffusivities [11] of water and TBA vapors in the porous dried layer were calculated and plotted in Fig. 5. As expected, water being lighter than TBA by a factor of approximately 4, has a Knudsen diffivisity about two times larger than that of TBA. The binary bulk diffusivity [11] is affected by both temperature and total pressure, which are both varying during freeze drying. Given the low total pressure condition (see Fig. 4) and considering the effective collision diameters of water and TBA being $2.64 \AA$ [12] and $5.40 \AA$ [13], respectively, the binary bulk diffusivities were calculated [11] and are represented by solid curves in Fig. 6 where the total vapor pressures are also included (dashed curves) for reference. Even the lowest bulk diffusivity in the range occurng at $-8.3^{\circ} \mathrm{C}$ and $x_{\mathrm{TBA}} \approx 0.85$ is still $50-100$ times greater than the Knudsen diffusivities of water and TBA vapors.

Enthalpically, water and TBA have very similar heats of sublimation, both around 51 $\mathrm{kJ} / \mathrm{mol}[10,14]$, so their frozen binary mixtures also require a similar heat of sublimation [10]. This fact likely reduces the number of variables to control when devising an optimal heating strategy for the freeze drying process. In this respect, the system temperature cannot exceed $-8.3{ }^{\circ} \mathrm{C}$ as it is the lowest melting temperature of the frozen water-TBA mixtures over the entire compositional range, which is also an eutectic point [7] occurring at $17.8 \mathrm{wt} \%$

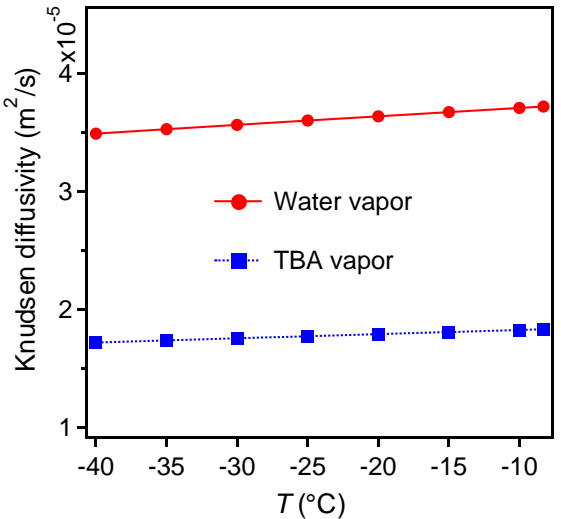

Fig. 5 Knudsen diffusivity of water and TBA as a function of temperature

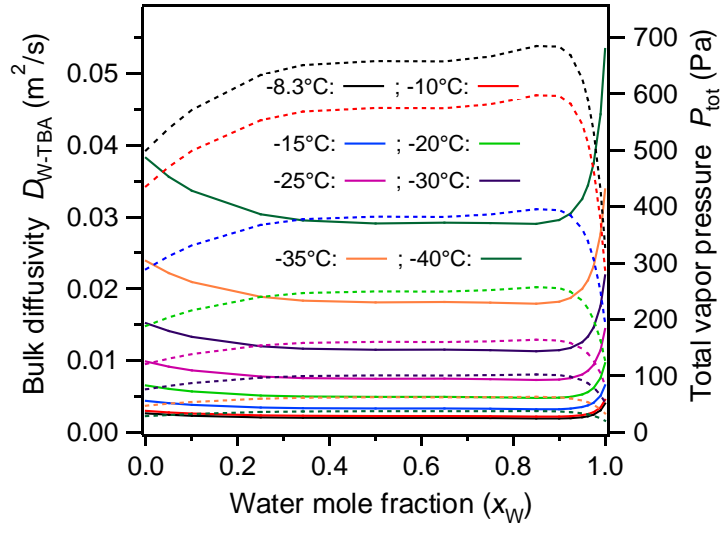

Fig. 6 Binary bulk diffusivity and total vapor pressure as a function of temperature and water mole fraction in the binary frozen solid mixture 
TBA $\left(x_{\mathrm{TBA}}=0.05\right)$. Similar heats of sublimation also attest, from the viewpoint of the Clausius-Clapeyron equation, the constantly higher pressures of TBA vapor over those of water vapor, and hence faster removal of TBA than water could occur during the primary drying stage of the freeze drying process. But the above data still point to water being a major process controlling species with respect to system constraints that have to be satisfied in the freeze drying process of pharmaceutical water solutions with TBA as a co-solvent.

\section{Conclusions}

Due to the limitations of current experimental techniques, a new class of physicochemical mathematical models is needed in order to enable rational physical and quantitative evaluation of the freeze drying process of pharmaceutical water solutions involing tertiary butyl alcohol (TBA) as a co-solvent. The construction and solution of such models require knowledge of the vapor pressures of water and TBA in equilibrium with their binary frozen solid mixtures, but these pressure data were not available to date. A thermodynamic approach based on the UNIFAC method coupled with the Dortmund parameters was developed to provide the needed pressure data as a function of temperature and of the mole fraction of the binary frozen solid mixture of water and TBA. The results have further been correlated into analytical expressions using a NRTL type model function. The results indicate the TBA vapor pressues to be constantly higher than those of water vapor and this leads to higher total pressure gradients in the porous dried layer of the product that could increase the convective mass transfer rates and support the experimental observation that TBA is removed relatively faster than water during the primary drying stage. When the bulk and Knudsen diffusion coefficients were evaluated under the temperatures and total vapor pressures that could be encountered in freeze drying, the former was found to be at least 50-100 times higher in magnitude than the latter. But the higher in magnitude total pressures of the binary gas mixtures of water and TBA which are due to the significantly larger vapor pressures of TBA, reduce the magnitude of the bulk diffusion coefficient. Furthermore, the Knudsen diffusion coefficient of TBA vapor is significantly smaller than that of water vapor. These effects on the magnitudes of the diffusivities could affect the mass transfer rates due to bulk and Knudsen diffusion during the primary and secondary drying stages. To ascertain the relative importance of the competing mass transport mechanisms of convection and bulk and Knudsen diffusion on the drying rates, one has to construct and solve an unsteady-state and spatially multi-dimensional freeze drying model that accounts for the effects of TBA presented here, while the thermodynamic expressions developed and presented in this work are a necessary component of the mathematical physics structure of such a model.

\section{References}

[1] Rey, M.; May, J.C. Freeze Drying/Lyophilization of Pharmaceutical and Biological Products; Marcel Dekker: New York, 2004.

[2] Millman, M.J.; Liapis, A.I.; Marchello. J.M. An analysis of the lyophilization process using a sorption - sublimation model and various operational policies. AIChE Journal 1985, 31 (10), 1594-1604.

[3] Sadikoglu, H.; Liapis, A.I. Mathematical modelling of the primary and secondary 
drying stages of bulk solution freeze drying in trays: Parameter estimation and model discrimination by comparison of theoretical results with experimental data. Drying Technology 1997 (3-4), 15, 791-810.

[4] Liapis, A.I.; Bruttini, R. Exergy analysis of the freeze drying of pharmaceuticals in vials and trays. International Journal of Heat and Mass Transfer 2008, 51, 3854-3868.

[5] Liapis, A.I.; Bruttini, R. A mathematical model for the spray freeze drying process: The drying of frozen particles in trays and in vials on trays. International Journal of Heat and Mass Transfer 2009, 52, 100-111.

[6] Bruttini, R; Liapis, A.I. The drying rates of spray freeze drying systems increase through the use of stratified packed bed structures. International Journal of Heat and Mass Transfer 2015, 90, 515-522.

[7] Kasraian, K.; DeLuca, P.P. Thermal analysis of the teriary butyl alcohol-water system and its implications on freeze drying. Pharmaceutical Research 1995, 12 (4), 484-490.

[8] Gmehling, J.; Li, J.; Schiller, M. A modified UNIFAC model. 2. Present parameter matrix and results for different thermodynamic properties. Industrial \& Engineering Chemistry Research 1993, 32 (1), 178-193.

[9] Oettin, F.L. The heat capacity and entropy of 2-methyl-2-propanol from 15 to $330 \mathrm{~K}$. Journal of Physical Chemistry 1963, 67 (12), 2757-2761.

[10] Bogdani, E.; Daoussi, R.; Vessot, S.; Jose, J.; Andrieu, J. Implementation and validation of the thermogravimetric method for the determination of equilibrium vapour pressure values and sublimation enthalpies of frozen organic formulations used in drug freeze-drying processes. Chemical Engineering Research and Design, 2011, 89 (12), 2606-2612.

[11] Geankoplis, C.H. Transport Processes and Separation Process Principles; Prentice Hall: Upper Saddle River, New Jersey, 2003.

[12] Hirschfelder, J.O.; Curtiss, C.F.; Bird, R.B. Molecular Theory of Gases and Liquids; Wiley: New York, 1954.

[13] Westgate, P.J.; Ladisch, M.R. Sorption of organics and water on starch. Industrial \& Engineering Chemistry Research 1993, 32 (8), 1676-1680.

[14] Murphy, D.M.; Koop, T. Review of the vapour pressures of ice and supercooled water for atmospheric applications, Quarterly Journal of the Royal Meteorological Society 2005, 131 (608), 1539-1565. 